\title{
Effect of vesicoureteric reflux on renal growth in children with urinary tract infection
}

\author{
JEAN M SMELLIE, DAVID EDWARDS, I C S NORMAND, AND NINA PRESCOD \\ Department of Paediatrics and Department of Radiology, University College Hospital, London, and \\ Department of Child Health, University of Southampton, Southampton General Hospital, Hampshire
}

SUMMARY Renal growth was studied for periods ranging from 2 to 22 years (average 9.4) in 70 children who presented between 2 weeks and 12 years of age with urinary tract infection and who were found to have vesicoureteric reflux. They were managed on a conservative regimen of continuous prophylaxis and regular, complete voiding. Renal growth over the entire period of observation was normal in relation to the child's growth in height in 100 of the 111 kidneys originally drained by refluxing ureters. During the time that reflux was known to be still present, renal growth was within normal limits in 101 kidneys. Renal growth was impaired in 11 kidneys. Ten of them were exposed to a recurrence of urinary tract infection. The eleventh child had an unproved symptomatic infection. Seven of these kidneys were also already scarred and 4 had gross reflux. In 2 of these children a fresh scar developed, one in a previously normal kidney. Because of the tendency for reflux to disappear, the renal growth during the first 2 years of observation was also analysed. A significant association was found between impaired growth and, independently, infection and renal scarring. No independent association was found between impaired growth and the severity of reflux. Small kidneys tended to grow at the expected rate. If growth was compared in pairs of kidneys with unilateral reflux, no difference in rate could be found if the kidneys were unscarred, but a significant difference was found if the refluxing ureter drained a scarred kidney; this tended to grow slowly, and the unscarred non-refluxing kidney tended to accelerate in growth. The small group of kidneys which grew least well had established severe scarring associated with severe persisting vesicoureteric reflux and each had a further infection during the period of observation, although no rise in plasma creatinine concentration was observed in any of these children. It is in such children that the results of a controlled comparison of medical and surgical management will be of greatest value.

Normal renal growth is a well-known indication of a healthy kidney, and in children it has a linear relationship with the child's height. ${ }^{1}$

In the early 'sixties, when fresh interest in vesicoureteric reflux (VUR) had followed the recognition of its association with chronic atrophic pyelonephritis and obstructive atrophy, we planned to study the growth of the kidneys in children who had VUR but in whom urinary tract infection was largely prevented by low-dosage antibacterial prophylaxis and regular complete voiding.

The outcome of reflux in the first 75 children with infection of the unobstructed urinary tract found to have VUR and managed in this way has already been published. ${ }^{2}$ Reflux disappeared spontaneously with time in $85 \%$ of undilated ureters and in $41 \%$ of those with dilatation. Fresh scarring developed in only 2 kidneys. Detailed observations on renal growth and scarring are now presented in 70 of these children who have been followed up for periods of 2 to 22 years.

\section{Children and methods}

Methods. Children presenting with urinary tract infection and found on micturating cystography (MCU) at least 2 weeks after eradication of infection to have VUR without outflow obstruction were given low-dosage prophylactic antibacterial drugs for as long as the reflux persisted, sulphonamide or nitrofurantoin at first and more recently cotrimoxazole. They were encouraged to pass urine regularly, frequently (at least every 3 hours), and completely, with double micturition at bedtime, and to avoid faecal overloading by regulation of bowel habits. Their progress was monitored at 
3-monthly intervals by urine culture, clinical assessment, and measurement of height, and of blood pressure if there was renal scarring. If a child was unwell or if fever, abdominal pain, or urinary symptoms occurred there were additional visits or urine dipslide cultures were made.

Renal length measurements were made on serial one- or two-film standard intravenous urograms (IVUs) taken at 2-yearly intervals as previously described, ${ }^{2}$ and related to the child's height using Hodson's standards. ${ }^{1}$ The renal parenchymal thickness was also assessed (taking particular note of the medial aspects of the polar regions). The MCU was repeated 2-yearly until reflux disappeared and then one year later to confirm its cessation. If it had reappeared, a limited MCU was again repeated after 2 years. To reduce irradiation the interval between cystograms was occasionally extended to 3 years if renal growth was satisfactory and if a significant residue was still present on double micturition.

Children. Renal growth measurements were possible over periods of 2 and up to 22 years in 70 of the first 75 children who were managed on this regimen ( 3 children with duplex systems, 1 with a horseshoe kidney, and 1 poor attender were excluded). There were 16 boys and 54 girls with ages on presentation ranging from 2 weeks to 12 years, $60 \%$ being aged 5 or less. Plasma creatinine concentration was within the normal range. Two girls were hypertensive.

VUR was present in 111 ureters and was bilateral in 41 children and unilateral in 29. Seven children had siblings with VUR who had attended the clinic with symptomatic infection. Seventeen children had scarring of at least one kidney. In 5 children treated surgically, renal growth was included up to the time of operation. (In one boy with a scarred kidney developing a fresh scar, this period was only 2 years; in the others, medical management and observation extended up to 7 years.)

Analysis of results. Renal growth measured over the entire period was related to the initial severity of reflux, to recurrence of infection during the observation period, and to the presence of renal scarring. Renal growth was also compared in pairs of kidneys with unilateral reflux, and longitudinally in kidneys before and after reflux stopped.

Assessment of the effect of different factors-such as infection or severity of reflux-on renal growth is complicated by the tendency for reflux to disappear spontaneously, by the different ages at presentation, by the occurrence of infection in relation to the timing of IVUs from which measurements of renal growth were obtained, and by the extent, location, and continuing contraction of renal scars. The technical problems of measuring renal size on IVU are also greater in scarred kidneys as there may be focal reduction in dye concentration or superimposition of more than one outline, as may occur with polar scars. The morphology of the individual kidney remains fairly constant so that evaluation may be simpler on serial than on individual assessments.

In view of these complexities, renal growth was also analysed over approximately 2-year periods. This growth was related to the severity of VUR at the start of each period, to the occurrence of infection during the month preceding the first IVU and up to one month before the second IVU of each 2-year period, as well as to the presence of scarring.

The number of observations in each patient depended upon how long reflux persisted, and ranged from one period (of 2 years) to 5 such periods. Repeated observations in the same patient were assumed to be independent only as far as the variables of infection and severity of reflux were concerned. Because of the permanence of scarring, the individual 2-year periods affecting kidneys with and without scars were analysed independently.

Terminology. Renal growth was regarded as slow during the period of observation if there was a discrepancy of at least $1 \mathrm{~cm}$ between the observed and the expected increase in renal length. When individual 2-year periods of growth were compared, growth was regarded as slow if the kidney increased in length by less than half that expected from the child's height during the 2-year period.

Growth was considered to be accelerated when the observed increase in length exceeded that expected overall by $1 \mathrm{~cm}$, or in a 2-year period by more than half that expected.

Reflux was graded as in previous reports. Grade 1 reflux was minimal, grades 2 and 3 extended up to the kidney without dilatation, grade 2 on voiding only. Grade 4 included all reflux with dilatation of the ureter or renal pelvis. ${ }^{2}$

\section{Results}

Seventy children were followed clinically for 658 child years, average 9.4 years per child, and radiologically over 574 child years.

Clinical and microbiological findings. Compliance in the regimen was stated by the patients to be good, both in taking prophylaxis and in adhering to the programme of voiding. Follow-up attendance was regular and urine samples collected at random clinics were tested for antibacterial activity which was present in $75-90 \%$ of children at different 
clinics. No appreciable side effect was seen from any drug used. Somatic growth was satisfactory. Malignant hypertension was well controlled in 2 girls. Two other children, both with unilateral renal scarring, developed hypertension sufficient to require hypotensive treatment. One was a 15 -year-old boy, in whom hypertension developed 4 years after successful ureteric reimplantation for $\mathrm{VUR}^{3}$ and the other, a 16-year-old girl, developed hypertension 6 years after spontaneous cessation of bilateral VUR.

Twenty-one children (6 of them boys) had at least one infection during the study (a recurrence rate of 1 per 18 years of observation). There were 36 infections, 9 of them single, exposing 30 kidneys to an episode of reinfection while reflux was present. One-third were symptomatic and developed between routine visits. The remainder were symptomless and were detected on routine culture. The organisms isolated were Escherichia coli $(90 \%)$, Klebsiella sp. $(3 \%)$, Proteus mirabilis (4\%), and Streptococcus faecalis $(2 \%)$.

Plasma creatinine concentration remained within normal limits in all children, but no differential renal function studies were made.

\section{Renal growth.}

Overall growth in 111 kidneys

Renal growth was observed in 111 kidneys all initially drained by refluxing ureters and during a total of 941 kidney years. It appeared normal in 100 $(90 \%)$ kidneys, the increase in length being within $1 \mathrm{~cm}$ (about $1 \mathrm{SD}$ ) of that expected from the child's height.

Relation to scarring. Nineteen refluxingureters drained kidneys which were already scarred on presentation. In 8 of these there were at most 2 scarred areas; in 7 scarring was fairly widespread and in 4 it was of the more uniform obstructive atrophic type often associated with gross reflux. ${ }^{4}$ Ninety-six per cent of the unscarred and $12(63 \%)$ of the scarred kidneys grew normally (Table 1 ).

Relation to infection. Two-thirds of the 30 kidneys exposed to reinfection grew normally, but 10 of the 11 kidneys in which growth was impaired were

Table 1 Renal growth in 111 kidneys drained by

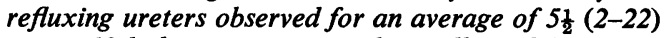
years, 19 kidneys were scarred initially and 1 scar developed during observation

\begin{tabular}{lllc}
\hline Renal growth & No scar & Renal scars & Total \\
\hline Normal & 88 & 12 & 100 \\
Slow & $4^{*}$ & 7 & 11 \\
\hline
\end{tabular}

* Fresh scar.

$\chi^{2}=18 \cdot 6$, df $1, P<0.001$. among those with a recurrence of infection (Table 2). There was a significant difference in growth between those renal units exposed to infection during the study and those remaining sterile. Five children had more than 1 infection exposing 8 kidneys to a repeat infection, but in only 1 of these was growth impaired overall.

Relation to severity of reflux on presentation (Table 3). Overall renal growth was impaired in only 5 of 93 kidneys drained by ureters which were undilated on presentation (grades 1-3), but one-third of the 18 kidneys drained by dilated ureters showed some impairment of growth. Five of these 6 slow-growing kidneys associated with grade 4 reflux were already scarred, and the sixth developed a scar; a reinfection occurred in all 6 . Thus it was not possible to establish a significant relationship between impaired growth and severe VUR without infection or scarring.

The other 12 kidneys ( 3 of them scarred at first) grew normally. Two young children aged 2 weeks and 18 months had bilateral grade 4 reflux which stopped at ages 10 and 5 years; there were no further infections and all 4 kidneys remained within normal limits in length. The infant boy had a small polar scar at age 1 which did not extend.

Slow growth. Seven of the 11 kidneys in which overall growth was slow had established scars, and an eighth developed a new scar in a previously normal kidney. Four were in boys, all with grade 4 reflux; 4 were small at the start. Reflux was severe in 6 , extended up to the kidneys without dilatation in 3 , and was minimal in 2. It persisted in 6 ureters (but has now

Table 2 Renal growth in 111 kidneys with vesicoureteric reflux related to recurrence of infection during 791 kidney-years of observation

\begin{tabular}{lllc}
\hline Renal growth & Infection & No infection & Total \\
\hline Normal & 20 & 80 & 100 \\
Slow & 10 & 1 & 11 \\
\hline
\end{tabular}

$\chi^{2}=25 \cdot 3$, df $1, P<0 \cdot 001$.

Table 3 Renal growth in 111 kidneys with vesicoureteric reflux, related to grade of reflux on first diagnosis

\begin{tabular}{llll}
\hline Renal growth & \multicolumn{2}{l}{ Vesicoureteric reflux } & Total \\
\cline { 2 - 3 } & Grades 1-3 & Grade 4 & \\
\hline Accelerated or normal & 88 & $12^{*}$ & 100 \\
Slow & 5 & $6+\ddagger$ & 11 \\
\hline
\end{tabular}

$\chi^{2}=13 \cdot 20$, df $1, P<0.001$.

*3 kidneys were scarred, +5 kidneys were scarred. $¥$ All 6 were exposed to infection. 
been corrected surgically in 4 of them). Infection confirmed by culture recurred in 10 of the 11 children. The last kidney without proved reinfection was small and scarred; there was VUR without dilatation, which stopped spontaneously. This girl had controlled hypertension and a suspected but unproved urinary tract infection.

Relation to age and sex (Table 4). Half of the 70 children ( 5 boys and 30 girls) were at least 5 years at diagnosis of VUR, and 11 of the 16 boys presented under age 5 years. Although there was a tendency for boys with VUR to present earlier than girls and for them to have a slightly higher proportion of scarred kidneys, no significant relationship was found between renal growth and the age of presentation or sex. Both fresh scars developed in children older than 5 years, one in a boy first seen at age 4 .

Small kidneys. On presentation 27 kidneys were $0.5-4.5 \mathrm{~cm}$ shorter than expected. During conservative management, all but 4 of these grew normally or slightly faster than expected whether they were scarred or not. These $\mathbf{4}$ had scarring of the obstructive atrophy type and persisting severe reflux with dilatation, and all had recurrences of infection.

Kidneys which were small initially tended to remain small, but grew at the expected rate unless scarring was severe.

Relation of renal growth to the presence of reflux. Reflux disappeared during the study, with confirmation by MCU at least 1 year later, in $55(79 \%)$ of these 70 children and in $92(83 \%)$ of the 111 refluxing ureters. Reflux persisted or was corrected surgically in 15 children and 19 ureters. Eight ureters, 6 with grade 4 reflux, were reimplanted in 5 children. In 8 further ureters, minimal reflux was still present on the last cystogram although in 3 of them (3 patients) this has not been repeated for at least 5 years.

Renal growth during reflux. A total of 574 years of renal growth was followed radiologically while reflux was known to be present, the longest individual period being 10 years. Renal growth assessed during

Table 4 Relationship of age and sex to renal growth in 111 kidneys with vesicoureteric reflux

\begin{tabular}{|c|c|c|c|c|c|}
\hline \multirow[t]{2}{*}{ Renal growth } & \multicolumn{2}{|c|}{ Age $0-4$ years } & \multicolumn{2}{|c|}{ Age 5-12 years } & \multirow[t]{2}{*}{ Total } \\
\hline & Boys & Girls & Boys & Girls & \\
\hline $\begin{array}{l}\text { Normal } \\
\text { Slow }\end{array}$ & $\begin{array}{r}12 \\
3\end{array}$ & $\begin{array}{r}33 \\
4\end{array}$ & $\begin{array}{l}6 \\
1\end{array}$ & $\begin{array}{r}49 \\
3\end{array}$ & $\begin{array}{r}100 \\
11\end{array}$ \\
\hline
\end{tabular}

Renal growth was not related to age at presentation $\left(\chi^{2}=1 \cdot 38\right.$, df $1, \mathrm{NS})$ either in boys or girls. this time was within the defined normal limits in $101(91 \%)$ kidneys.

Unilateral reflux. In 29 children VUR was unilateral, making possible a comparison of renal growth in paired kidneys with and without reflux. In 20 children both kidneys were structurally normal and remained so, although in 3 there was an initial discrepancy in length between them of more than $1 \mathrm{~cm}$. The mean renal lengths initially were not significantly greater than that expected in relation to the child's height, $0.02 \mathrm{~cm}$ in the refluxing and 0.08 $\mathrm{cm}$ in the non-refluxing kidney. In 8 children the kidneys drained by the refluxing ureter were scarred; in the ninth a scar developed. Reflux stopped spontaneously in all 20 ureters draining unscarred kidneys and in 6 of the 9 draining scarred kidneys. Two ureters were reimplanted and no recent cystogram has been carried out in the ninth patient.

The growth rate was similar in the 20 pairs of unscarred kidneys whether or not reflux was present, there being a slight increase in length over that expected of $0.16 \mathrm{~cm}$ (range -0.8 to $+1 \cdot 2$ ) and $0.15 \mathrm{~cm}$ (range -0.8 to $+1 \cdot 3$ ) per kidney respectively in the 2 groups, not a significant difference.

In the 9 children in whom the refluxing ureter drained a scarred kidney ( 8 of which were smaller by $0 \cdot 8-5 \cdot 3 \mathrm{~cm}$ than the contralateral non-refluxing kidney), the mean growth was $0.18 \mathrm{~cm}$ (range +0.3 to $-1 \cdot 3$ ) less than expected in the scarred kidney, and greater than that expected by an average of $1.03 \mathrm{~cm}$ (range 2.2 to -1.2 ) in the unscarred kidney, indicating some hypertrophy of the normal kidney. Seven of the 9 unscarred kidneys had an increase of $0 \cdot 3-2 \cdot 2 \mathrm{~cm}$ over that expected; one was $0.2 \mathrm{~cm}$ and the other, a boy with hypertension, was $1.2 \mathrm{~cm}$ less than expected. This tendency to hypertrophy of the normal non-refluxing kidney and impaired growth of the scarred kidney with VUR was confirmed by a paired $t$ test, the mean difference in growth being $1.21 \pm 0.33 \mathrm{~cm}(t=3 \cdot 7$, df 8 , $P<0 \cdot 01$ ).

Renal growth after reflux stopped. Renal growth was measured in 52 children during intervals of 1 to 14 years after reflux stopped, a total of 367 child years. Reflux stopped spontaneously in 49 of these children (80 ureters) and was corrected surgically in 3 (4 ureters). Of the 80 kidneys in children treated medically, 66 were unscarred and 14 were scarred. Growth was normal in 75 , it was impaired in 3 unscarred kidneys, and was $0.5 \mathrm{~cm}$ greater than expected in 2 scarred kidneys during the first 2 years after reflux stopped. Longer observations were made in 56 kidneys. Growth was normal in 48 , it was impaired in 2 unscarred and 2 scarred kidneys, and 
was accelerated in 2 unscarred and 2 scarred kidneys (no conclusion thus being possible).

Of the surgically-treated children, satisfactory growth without acceleration occurred in 3 of the 4 kidneys.

\section{Growth in 2-year periods in 111 kidneys}

In order to differentiate the effects of infection, severity of reflux, and renal scarring, renal growth was measured in the 111 kidneys during 255 periods of about 2 years while VUR was present. It was impaired in $41(16 \%)$ and normal in 214 . Fifty-two of the 2-year observations were made in scarred kidneys showing slow growth in $29 \%$, and 203 were in normal kidneys and growth was impaired in $13 \%$.

Urinary tract infection occurred within the month preceding the first IVU or up to one month before the second IVU in 89 2-year periods, and renal growth was impaired in $34 \%$ of them. Urinary infection occurred in $31(76 \%)$ of the 41 periods with impaired growth (Table 5). In 52 periods observed in scarred kidneys, growth was slow in $11(61 \%)$ of the 18 when infection occurred, and was slow in only $4(12 \%)$ of the 34 when there was no infection.

When the effect of severity of reflux was examined, it was found that renal growth was impaired in only $13 \%$ of kidneys with reflux graded $1-3$ at the start of the observation period compared with $28 \%$ with grade 4 , but this association was complicated by the additional presence either of infection or renal scarring.

It became apparent in making this analysis that the ureters in which VUR was most likely to persist were those with gross reflux, draining scarred kidneys, so that there was a disproportionate representation of these kidneys in the analysis of the 2-year growth periods.

\section{First 2-year growth period-111 kidneys}

Renal growth was therefore compared in all 111 kidneys during the first 2-year period. Growth was slow in 28 and normal in 83 , and a significant association was found between impaired growth and infection $\left(\chi^{2}=10.71\right.$, df $\left.1, P<0.01\right)$ and also renal scarring $\left(\chi^{2}=5 \cdot 96\right.$, df $\left.1, P<0.05\right)$ but not severity of reflux $\left(\chi^{2}=0 \cdot 75\right.$, df $\left.1, \mathrm{NS}\right)$. When renal growth

Table 5 Renal growth in 255 2-year periods in 111 kidneys with vesicoureteric reflux showing relationship between renal growth and infection occurring during, or up to 1 month before, the first intravenous urograms of each growth period

\begin{tabular}{llll}
\hline Renal growth & No infection & Infection & Total \\
\hline Normal & 156 & 58 & 214 \\
Slow & 10 & 31 & 41 \\
\hline
\end{tabular}

$\chi^{2}=35 \cdot 6$, df $1, P<0.001$. was related to scarring and corrected for infection and severity of reflux only the scarred kidneys exposed to infection were significantly slowed $\left(\chi^{2}=4.43\right.$, df $\left.1, P<0.05\right)$. A similar correction for renal growth related to severity of reflux confirmed the lack of association between them. When renal growth was examined in relation to urinary infection, a close association was found in unscarred kidneys $\left(\chi^{2}=6.94\right.$, df $\left.1, P<0.01\right)$ and in kidneys with undilated refluxing ureters (grades $1-3)\left(\chi^{2}=6 \cdot 68\right.$, df $1, P<0.01$ ) but the numbers of scarred kidneys and those with grade 4 reflux were too few for satisfactory analysis.

Renal scarring. As already stated, one scar developed after a $S$. faecalis infection in the normal kidney drained by a dilated, refluxing ureter of a 9-year-old boy ${ }^{4}$ and a fresh scar developed after a symptomatic $E$. coli urinary tract infection in a 9-year-old boy with an established polar scar, grade 3 reflux, and a large paraureteric saccule. ${ }^{3}$ No scarring was seen to develop in any kidney without a recurrence of infection.

\section{Discussion}

In this study we planned to observe the effect in the unobstructed urinary tract of sterile VUR on renal growth, since renal growth without scarring is regarded as a reliable indicator of normality. Our first finding however was that in many children VUR became less severe and stopped. Nevertheless it was quite clear that while VUR persisted, normal growth could be expected in unscarred, uninfected kidneys whatever the severity, and this was also true in most kidneys with moderate scarring. In kidneys which were severely scarred with little normal parenchyma remaining, growth was invariably impaired; these kidneys were generally drained by dilated ureters with continuing gross VUR. Reinfection was also more likely in these kidneys.

Recurrence of urinary tract infection was not entirely prevented by the medical programme of prophylaxis and complete voiding, and this allowed a comparison of the effect of sterile reflux with that when the urine was infected. This showed the most important association of reflux and impaired renal growth to be infection. Ten of the 11 kidneys with growth impairment had reinfections during followup, and infection was associated with two-thirds of the 2-year periods which showed impaired growth. Infection also preceded the development of the 2 fresh scars which were seen, and no scarring developed in any uninfected refluxing urinary tract.

It was also clear that kidneys which on presentation were shorter in length than expected, whether 
through scarring or not, were likely to grow at the expected rate but without necessarily catching up. In children with unilateral VUR the presence of reflux did not affect the rate of renal growth provided there was no scarring. If the unilateral VUR drained a scarred kidney however, growth was generally slower than on the opposite side which tended to show accelerated growth and possible compensatory hypertrophy.

A standard medical regimen was used throughout the study and the good compliance and low recurrence rate (which improved after the introduction of co-trimoxazole prophylaxis) ${ }^{5}$ was probably largely attributable to continuity of supervision and care.

Admission to hospital was discouraged because of the increased likelihood of acquiring a resistant bowel flora and the risk of further urinary tract infection. In practice management was almost entirely on an outpatient basis. No appreciable increase in plasma creatinine occurred in any child, but 2 children developed hypertension during follow-up.

Methods have recently been introduced for studying renal growth which relate renal length measurement to the height of L 1-3 on the IVU film. ${ }^{6}$ However, as we knew the height of each child throughout the observation period, we used this measurement as the reference standard for renal length. We applied both methods to the same series of IVUs and found the results for renal growth were similar, but that the use of the child's height measurement was simpler and showed less observer variation.

In many reports of the effect of surgical correction of VUR on renal growth, results have been presented as a mean which has not taken into account the child's age or the infection history; we did not note acceleration of renal growth after reflux stopped although it has been reported to follow the surgical correction of VUR. ${ }^{78}$ This might perhaps be because the point at which reflux stopped was less clearly demarcated in children managed medically. More important, the time when infection was prevented from reaching the kidney and the time when reflux ceased were dissociated in the medicallytreated children. Normal renal growth proceeded from the time reinfection was prevented, whereas ureteric reimplantation could prevent both VUR and the access of infection to the kidney simultaneously.

From this study it can be concluded that in children with VUR and without obstruction, renal growth is likely to be normal if the kidneys are unscarred and there is no recurrence of infection. Growth may be transiently impaired after an infection in the presence of reflux, and may be impaired if there is renal scarring. In general, the prognosis for renal growth is excellent. Renal scarring is permanent, and if it is present, hypertension may develop whether reflux is present, has stopped spontaneously, or has been corrected surgically. ${ }^{9}$ In the very small group in this study of children with gross, persisting VUR, without outflow obstruction, and with severe generalised scarring, there is a slightly increased tendency for infection to recur and the prognosis for renal growth is less good.

The numbers in this study in which renal growth was impaired are too few to draw firm conclusions, but point to the importance of identifying the specific factors in the human kidney which predispose to renal scarring. They also indicate the need for a controlled comparison of optimal medical and surgical treatment of the children with the most severe reflux without obstruction.

We thank Dr A Johnson, MRC Statistical Research and Services Unit, Sister March, and many medical and nursing colleagues for assistance, and Mrs M Bedford for secretarial help.

This work was financially supported by the National Kidney Research Fund, Beecham Research Laboratories, the Medical Research Council, and University College Hospital research funds.

\section{References}

1 Hodson C J. Reflux nephropathy: scoring the damage. In: Hodson J, Kincaid-Smith P, eds. Reflux nephropathy. New York: Masson, 1979: 29-47.

2 Edwards D, Normand I C S, Prescod N, Smellie J M. Disappearance of vesicoureteric reflux during long-term prophylaxis of urinary tract infection in children. $\mathrm{Br} \mathrm{Med} \mathrm{J}$ 1977; ii: 285-8.

3 Smellie J M, Normand I C S. Reflux nephropathy in childhood. In: Hodson J, Kincaid-Smith P, eds. Reflux nephropathy. New York: Masson, 1979: 14-20.

4 Smellie J M, Edwards D, Hunter N, Normand I C S, Prescod N. Vesico-ureteric reflux and renal scarring. Kidney Int 1975; 8: Supplement 4, S65-72.

5 Smellie J M, Grüneberg R N, Leakey A, Atkin W S. Long-term low-dose co-trimoxazole in prophylaxis of childhood urinary tract infection: clinical aspects. $\mathrm{Br} \mathrm{Med}$ $J$ 1976; ii: 203-6.

6 Eklöf O, Ringertz H. Kidney size in children: a method of assessment. Acta Radiol [ Diagn] (Stockh) 1976; 17: 617-25.

7 Willscher M K, Bauer S B, Zammuto P J, Retik A B. Renal growth and urinary infection following antireflux surgery in infants and children. J Urol 1976; 115: 722-5.

8 Atwell J D, Vijay M R. Renal growth following reimplantation of the ureters for reflux. Br J Urol 1978; 50: 367-70.

9 Wallace D M A, Rothwell D L, Williams D I. The long-term follow-up of surgically treated vesicoureteric reflux. BrJ Urol 1978; 50: 479-84.

Correspondence to $\operatorname{Dr} \mathbf{J}$ M Smellie, Paediatric Department, University College Hospital, Gower Street, London WC1E 6AU.

Received 4 August 1980 